\title{
Conservative cooperative spectrum sensing without CRC in CR network
}

\author{
Taiping Cui and Kyungsup Kwak ${ }^{\mathrm{a})}$ \\ Telecommunication Engineering Laboratory, Inha University \\ 100 Inha-ro, Nam-gu, Incheon 402-751, Korea \\ a)kskwak@inha.ac.kr
}

\begin{abstract}
Cooperative spectrum sensing can detect the primary signal more reliably via exploiting spatial diversity, which usually requires extra common reporting channel for cognitive radios to exchange local sensing decision. In this letter, we propose a conservative cooperative spectrum sensing scheme, which can utilize the licensed channel for transmitting local sensing decision with limited interference to primary users. The new scheme can save the common reporting channel without sacrificing ROC performance and mitigate total interference probability compared with conventional cooperative sensing scheme.
\end{abstract}

Keywords: cognitive radio, conservative cooperative spectrum sensing, common reporting channel

Classification: Terrestrial Wireless Communication/Broadcasting Technologies

\section{References}

[1] Y. Liang, Y. H. Zeng, E. Peh, and A. T. Hoang, "Sensing-throughput tradeoff for cognitive radio networks," IEEE Trans. Wireless Commun., vol. 7, no. 4, pp. 1326-1337, 2008.

[2] J. Ma, G. Zhao, and Y. Li, "Soft Combination and Detection for Cooperative Spectrum Sensing in Cognitive Radio Networks," IEEE Trans. Wireless Commun., vol. 7, no. 11, pp. 4502-4507, 2008.

[3] G. Ganesan and Y. Li, "Cooperative spectrum sensing in cognitive radio: part I: two user networks," IEEE Trans. Wireless Commun., vol. 6, no. 6, pp. 2204-2213, 2007.

[4] T. Chen, H. Zhang, M. D. Katz, and Z. Zhou, "Swarm Intelligence Based Dynamic Control Channel Assignment in CogMesh," Proc. IEEE Int. Conf. on Communications Workshops, May 2008.

[5] M. Ohta, T. Fujii, K. Muraoka, and M. Ariyoshi, "A novel power controlled sensing information gathering for cooperative sensing on shared spectrum with primary spectrum," Proc. Int. ICST Conf. on CROWNCOM, June 2011.

[6] I. F. Akyildiz, B. F. Lo, and R. Balakrishnan, "Cooperative spectrum sensing in cognitive radio networks: A survey," Physical Communication, vol. 4, no. 1, pp. 40-62, 2010. 


\section{Introduction}

In cooperative spectrum sensing $[1,2,3]$, common reporting channel (CRC) is commonly assumed and utilized by cognitive radio (CR) users to report their local sensing decisions to a fusion center or share the sensing results with neighboring nodes. Without a CRC, previous cooperative sensing schemes can not provide channel for reliable communications between CRs. In [4], a swarm intelligence-based approach to solve the common control channel assignment probleme is proposed. The licensed channel can be used for gathering sensing information based on OF-SIG [5], Generally, a CRC needs extra channel allocation and increases complexity [6]. Also it may deteriorate the ROC performance and induce more interference to primary user (PU).

Motivated by the feasiblity of using licensed channel as CRC, in this letter, we first propose a conservative cooperative sensing strategy to report and utilize the licensed channel. In addition, we investigate the transmission power to achieve a reliable cooperation. Finally, we analyze the interference probability induced by the proposed spectrum sensing scheme.

\section{System model}

For PU network, we assume that it consists one transmitter and several receivers, e.g., a TV radio station and many TV radio receivers. For CR network, we investigate a network with two CRs operating in a fixed TDMA mode. Each CR can execute spectrum sensing during its sensing time slot and $\mathrm{CR} 1$ sends its local sensing decision to CR0 periodically to improve the detection performance of CR0. We will present the cooperation procedure in detail in the following section.

The local spectrum sensing is accomplished by energy detection for implementation simplicity and let $\tau$ be the identical sensing time for each CR. Suppose that the received signal at CR is sampled at sample frequency $f$. Then the number of samples $M$ is the maximum integer not great than $\tau f$, i.e., $M=\lfloor\tau f\rfloor$. The accumulated energy $Y_{i}$ for the $i$ th $C R$ can be expressed as

$$
Y_{i}= \begin{cases}\frac{1}{M} \sum_{k=1}^{M}\left|n_{i}(k)\right|^{2}, & H_{0}, \\ \frac{1}{M} \sum_{k=1}^{M}\left|s(k)+n_{i}(k)\right|^{2}, & H_{1},\end{cases}
$$

where $n_{i}(k)$ is the complex Gaussian noise with mean zero and variance $\sigma_{n}^{2}$, and $s(k)$ is the PU signal. $H_{0}$ and $H_{1}$ denote the hypotheses corresponding to the absence and presence of the PU signal, respectively.

The purpose of the energy detection is to make a reliable decision between $H_{0}$ and $H_{1}$ based on the observation $Y_{i}$. Denote $\gamma_{i}=\frac{\sum_{k=1}^{M}|s(k)|^{2}}{M \sigma_{n}^{2}}$ as the received signal-to-noise ratio (SNR) of the PU signal measured at $\mathrm{CR} i$, under the hypothesis $H_{1}$. Then the probability of false alarm $P_{f, i}$ and the probability of missed detection $P_{m, i}$ can be approximated by [1]

$$
P_{f, i}=Q\left(\left(\frac{\lambda}{\sigma_{n}^{2}}-1\right) \sqrt{M}\right)
$$




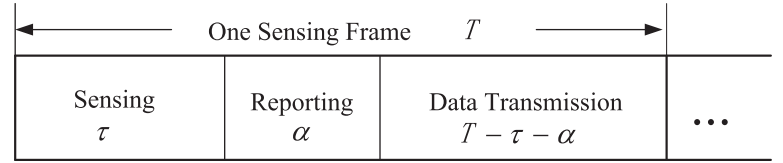

Fig. 1. Frame structure of cooperative spectrum sensing.

$$
P_{m, i}=1-Q\left(\left(\frac{\lambda}{\sigma_{n}^{2}}-\gamma_{i}-1\right) \sqrt{\frac{M}{1+2 \gamma_{i}}}\right)
$$

where $\lambda$ is the corresponding decision threshold and $Q(x)=\frac{1}{\sqrt{2 \pi}} \int_{x}^{\infty} e^{-\frac{t^{2}}{2}} d t$.

\section{Conservative cooperative spectrum sensing without com- mon reporting channel}

\subsection{Conservative cooperative strategy}

Fig. 1 shows the frame structure of cooperative spectrum sensing. Besides the previous defined sensing duration $\tau$, suppose that the reporting duration is $\alpha$ and the frame duration is $T$. In the sensing slot $\tau$, each CR need to sense the licensed band and achieves its local sensing decision. For conventional cooperative scheme, CR1 will send its sensing result to CR0 in the reporting slot and cooperative decision will be obtained at CR0 via combination of two decisions. If there is no CRC, we consider the licesed channel for reporting local sensing decsion when it is vacant. Since there is no CRC, if PU signal is present, CR1 will cause interference to PUs when it reports its sensing decision to CR0. To reduce the reporting data, instead of all sensing result, CR1 sends one predefined flag packet (FP) to CR0 to announce that the PU channel is vacant only when its sensing energy is less than $\lambda_{s}$. On the other hand, when its sensing energy is larger than $\lambda_{s}$, CR1 will keep silent which will mitigate the potential interference to PUs. For a target false alarm probability $P_{f, s}$, from (2), the threshold can be determined by

$$
\lambda_{s}=\left(1+\frac{1}{\sqrt{M}} Q^{-1}\left(P_{f, s}\right)\right) \sigma_{n}^{2} .
$$

The reasons why we term conservative are that we utilize the PU channel in a conservative manner and offer the best protection to PUs. First, if CR0 makes its local decision that PU signal is present, it will not receive the cooperation data from CR1 and keep silent in the rest of the frame. Second, in order to reduce the interference caused by reporting, we employ a conservative reporting strategy: CR1 will cooperate only when $Y_{1} \leq \lambda_{s}$. Third, CR0 can reuse the PU channel for data transmission only when FP is correctly received by CR0. In other word, if there is no FP from CR0 or error occurs, CR0 will consider the presence of PU as the decision of CR1.

\subsection{Reporting reliability}

Without a CRC, it is unreasonable to assume that the reporting channel for the proposed cooperative sensing scheme is perfect since it may be subjected 
to fading and interference from PU. Due to the error induced by the imperfect channel, the FP from CR1 may not be correctly received by CR0. Let $P_{e}^{H_{j}}$ denote the average FP error, where $H_{j} \in\left\{H_{0}, H_{1}\right\}$. Then at CR0, the cooperative missed detection probability $Q_{m}$ and cooperative false alarm probability $Q_{f}$ are presented by, respectively:

$$
\begin{gathered}
Q_{m}=P_{m, 0} P_{m, 1}\left(1-P_{e}^{H_{1}}\right), \\
Q_{f}=1-\left(1-P_{f, 0}\right)\left(1-P_{f, 1}\right)\left(1-P_{e}^{H_{0}}\right) .
\end{gathered}
$$

For simplicity, we assume BPSK modulation with transmit power $P_{c}$ for FP. When PU signal is present, the average FP error is

$$
\begin{aligned}
P_{e}^{H_{1}} & =\mathbb{E}\left[Q\left(\sqrt{\frac{2\left|h_{c}\right|^{2} P_{c}}{\left(1+\gamma_{0}\right) \sigma_{n}^{2}}}\right)\right] \\
& =\frac{1}{2}\left[1-\sqrt{\frac{\gamma_{c} \sigma_{c}^{2}}{1+\gamma_{0}+\gamma_{c} \sigma_{c}^{2}}}\right],
\end{aligned}
$$

where $h_{c}$ is the channel fading coefficient between CR1 and CR0, and the expectation $\mathbb{E}[\cdot]$ is taken with respect to the squared magnitude of the fading coefficient $\left|h_{c}\right|^{2}$ and $\gamma_{c}=\frac{P_{c}}{\sigma_{n}^{2}}$. Note that random variable $\left|h_{c}\right|^{2}$ in (7) and (8) follows the exponential distribution with parameter $\frac{1}{\sigma_{c}^{2}}$.

When PU signal is absent, the FP error is given by

$$
\begin{aligned}
P_{e}^{H_{0}} & =\mathbb{E}\left[Q\left(\sqrt{\frac{2\left|h_{c}\right|^{2} P_{c}}{\sigma_{n}^{2}}}\right)\right] \\
& =\frac{1}{2}\left[1-\sqrt{\frac{\gamma_{c} \sigma_{c}^{2}}{1+\gamma_{c} \sigma_{c}^{2}}}\right] .
\end{aligned}
$$

If there is no interference from $\mathrm{PU}, \mathrm{FP}$ should be reliably received by CR0. It can be expressed as $P_{e}^{H_{0}} \leq \varepsilon_{t h}$, where $\varepsilon_{t h}$ is the system requirement of the FP error. Then the minimum average reporting power can be derived as

$$
\gamma_{c} \geq \frac{\left(1-2 \varepsilon_{t h}\right)^{2}}{4\left(\varepsilon_{t h}-\varepsilon_{t h}^{2}\right) \sigma_{c}^{2}}
$$

\subsection{Interference analysis}

When CR1 fails to detect PU signal, the following reporting step will interfere $\mathrm{PU}$ receivers. The interference probability induced by reporting from CR1 to CR0 can be describe as

$$
P_{I, R}=\frac{\alpha}{T} P_{m, 1},
$$

where the factor $\frac{\alpha}{T}$ is due to the fact that the reporting duration occupies $\frac{\alpha}{T}$ fraction of one sensing frame.

If $\mathrm{CR} 0$ can correctly receive the FP from CR1, it will transmit data in the rest of the frame, $T-\tau-\alpha$. The interference probability induced by data transmission from CR0 can be presented by

$$
P_{I, T}=\frac{T-\tau-\alpha}{T} P_{m, 0} P_{m, 1}\left(1-P_{e}^{H_{1}}\right),
$$


where the factor $\frac{T-\tau-\alpha}{T}$ is due to the fact that the data transmission duration occupies $\frac{T-\tau-\alpha}{T}$ fraction of one sensing frame.

Based on (10) and (11), the total interference probability induced by CR network can be written as

$$
\begin{aligned}
P_{I} & =P_{I, R}+P_{I, T} \\
& =\frac{\alpha}{T} P_{m, 1}+\frac{T-\tau-\alpha}{T} P_{m, 0} P_{m, 1}\left(1-P_{e}^{H_{1}}\right) \\
& =\frac{1}{2 T}\left[1-Q\left(\left(\frac{\lambda_{s}}{\sigma_{n}^{2}}-\gamma_{1}-1\right) \sqrt{\frac{M}{1+2 \gamma_{1}}}\right)\right][2 \alpha+(T-\tau-\alpha) \\
& \left.\times\left(1-Q\left(\left(\frac{\lambda_{s}}{\sigma_{n}^{2}}-\gamma_{0}-1\right) \sqrt{\frac{M}{1+2 \gamma_{0}}}\right)\right)\left(1+\sqrt{\frac{\gamma_{c} \sigma_{c}^{2}}{1+\gamma_{0}+\gamma_{c} \sigma_{c}^{2}}}\right)\right] .
\end{aligned}
$$

In practice, the reporting duration $\alpha$ is much shorter than data transmission duration $T-\tau-\alpha$. So the interference induced by data transmission $P_{I, T}$ dominates the total interference probability $P_{I}$. From (11), we can clearly see that the proposed scheme can take advantage of the FP error $P_{e}^{H_{1}}$ to decrease the interference probability. Thus our proposed scheme can mitigate the total interference and offer the best protection to PUs.

\section{Simulation results}

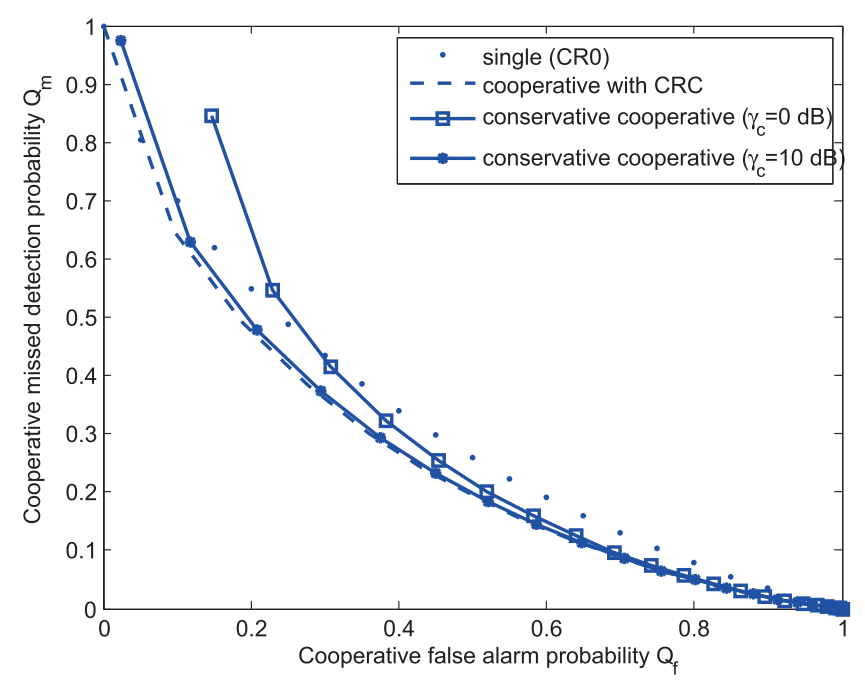

Fig. 2. ROC performance with $\gamma_{i}=-10 \mathrm{~dB}$.

In this section, we will present numerical results of the conservative cooperative sensing scheme without CRC for $\tau=10 \mathrm{~ms}, \alpha=1 \mathrm{~ms}, T=50 \mathrm{~ms}$, $f_{s}=5 \mathrm{kHz}, M=50$ and $\sigma_{c}^{2}=1$. We first show the ROC curves of the proposed and conventional cooperative sensing scheme. Fig. 2 illustrates the cooperative false alarm probability versus the cooperative missed detection probability. For conventional cooperative sensing, we assume an error free CRC for reporting sensing results from CR1 to CR0. When reporting power 


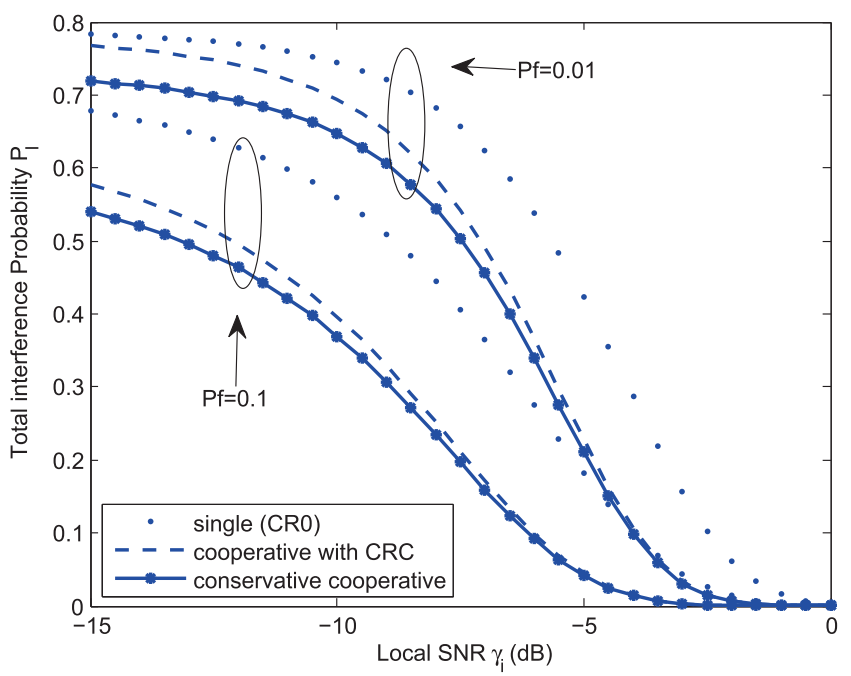

Fig. 3. The Local SNR versus the total interference probability with $\gamma_{c}=5 \mathrm{~dB}$.

is high, i.e., the FP can be reliably received by CR0 based on (9), our proposed scheme can achieve nearly identical performance compared with the conventional cooperative sensing scheme. However, when reporting power is low, due to FP error, the ROC performance of the proposed scheme is even worse than that of single user in low false alarm probability region.

Fig. 3 shows the total interference probability with increasing of the local SNR under different false alarm probability. From Fig. 3, one can see that cooperation can reduce total interference probability. Obviously, our conservative cooperative sensing scheme induces less total interference probability than that of the conventional cooperative sensing scheme. As expected, CRs cause more interference with a strict false alarm requirement. This is because that when false alarm is small, the selection threshold as given by (4) should be large, which results in low detection probability and thus increases the total interference probability.

\section{Conclusions}

In this letter, we have proposed a conservative cooperative sensing scheme without the CRC and analyzed the performance of the proposed scheme. Compared with the conventional cooperative sensing scheme with CRC, the proposed conservative cooperative sensing scheme can release the CRC without sacrificing ROC performance and mitigate the total interference probability to PUs.

\section{Acknowledgments}

This research was supported by the National Research Foundation of Korea (NRF) grant funded by the Korea government (MEST) (No.2010-0018116). 